\title{
Mirizzi syndrome
}

\author{
Md. Ibrarullah · Tapas Mishra • A P Das
}

Received: 2 October 2008 / Accepted: 4 November 2008

\begin{abstract}
Mirizzi syndrome is a complication of long standing cholelithiasis. In this, obstruction of the extrahepatic bile duct by stone/s in the Hartman's pouch or cystic duct (Mirrizi type I) may erode in to the bile duct forming cholecystobiliary fistula (Mirrizi type II). Altered biliary tract anatomy and the associated pathology make cholecystectomy, open or laparoscopic, a formidable undertaking. Awareness of this entity and its preoperative diagnosis is of paramount importance to avoid injury to the bile duct at surgery. Improper surgical procedures may lead to long-term stricture formation. The present article reviews the available literature on various aspect of this syndrome including its pathogenesis, diagnosis and recommended management guidelines.
\end{abstract}

Keywords Mirizzi syndrome - Obstructive jaundice . Cholecystobiliary fistula

\section{Introduction}

Mirizzi syndrome (MS) is defined as bile duct obstruction due to stone/s impacted in the Hartman's pouch or cystic duct. This entity was originally described by Kehr [1] and Ruge [2] and subsequently by P L Mirizzi [3]. At one time considered to be rare to merit reporting of isolated cases only [4-10], larger series on MS are now available in literature [11-17]. The reported incidence has ranged from 0.05 to $4 \%$ of all patients undergoing surgery for cholelithiasis [11-19]. These figures assume significance in view of the high prevalence of cholelithiasis and the high frequency of cholecystectomies performed world over. Considered a 'trap' in the surgery of cholelithiasis [11], presence of MS poses considerable challenge to the ingenuity of any surgeon operating on the biliary tract. At surgery the frozen calots triangle with or without bile duct erosion, presence of bilioenteric fistula in a sizable proportion of patients and its misinterpretation or coexistence of carcinoma gall bladder (GB) [11-16, 20-22], calls for extra caution on the part of operating surgeon. Inadequate or inappropriate surgery is fraught with the danger of injury to the bile duct $[11,16,18$ 19] or long-term stricture formation [12, 23]. In the era of minimal invasive surgery, this entity assumes further significance as the altered biliary tract anatomy may demand early conversion to open cholecystectomy in the majority. The present article is an attempt to review this entity in its various forms, there pathogenesis, diagnosis and management, particularly in light of modern technical advances.

\section{Pathogenesis and classification}

Md. Ibrarullah · T. Mishra $\cdot$ A. P. Das

Dept of Surgery \& Surgical Gastroenterology,

Hi-tech Medical College \& Hospital, Bhubaneswar - 751 010, Orissa, India

Md. Ibrarullah • T. Mishra $(\bowtie)$

Email:m_ibrarullah@yahoo.co.in tapasmishra08@Gmail.com
Biliary obstruction as described in the original MS was reported to have resulted from stones in the cystic duct leading to inflammation and spasm of the sphincter in the distal common hepatic duct (CHD); the entity was therefore called 'functional hepatic syndrome' [3]. However, subsequently the obstruction was proved to be purely mechani- 
cal, a result of external compression and inflammation by the stone in the Hartman's pouch or cystic duct. The pathogenesis was further clarified and the various components $[9,24-27]$ of this syndrome were identified as,

- Cystic duct parallel to CHD

- Impaction of a gallstone in the cystic duct or neck of the GB

- Partial mechanical obstruction of the CHD by the stone itself or the resulting inflammation

- Recurrent cholangitis or ultimately cholangitic cirrhosis due to partial obstruction.

Obstruction of common hepatic duct or common bile duct (CBD), both hence forth referred as common duct (CD), due to distended GB as in acute cholecystitis [28, 29] or tumours of the GB [30] or cystic duct [31-33] has also been described as variants of MS. However, in our opinion, it is preferable to avoid any such confusion in nomenclature and the syndrome should strictly refer to bile duct obstruction resulting from stone disease only.

Cholecystobiliary fistula (CBF), an internal biliary fistula between GB and the $\mathrm{CD}$ was first described by Puestow in 1942 [34] and by other authors subsequently [18, 35-38]. Various hypotheses have been proposed to explain its pathogenesis. The fistula was thought to represent a congenital anomaly with absent cystic duct [39]. The more plausible hypothesis, however, has been cystic duct occlusion due to stone and inflammation leading to distension of GB and approximation of its wall with that of CD; facilitated by the continued presence of a stone, pressure necrosis of the common wall and fistula formation $[6,12$, $14,40-43]$. McSherry views CBF as an advanced stage of MS, where the impacted stone has subsequently eroded into the CD forming a fistula. On this premise he classified MS into two types,

- Type I - bile duct obstruction by external compression and

- Type II - cholesystobiliary fistula [44].

Though CBF was further classified into two types depending on the presence or absence of cystic duct [11], we concur with Csendes et al [12] that such a classification is not relevant from management point of view. The latter author further subclassified the CBF according to the extent of circumferential erosion of $\mathrm{CD}$ and proposed a new classification. The various types according to this classification and there relative incidence are,

- Type I - obstruction of CD by stone/s impacted in the Hartman's pouch or the cystic duct $-11 \%$.

- Type II - cholecystobiliary fistula with erosion of less than $1 / 3$ of circumference of $C D-41 \%$.

- Type III - erosion of up to $2 / 3$ circumference of CD $-44 \%$.

- Type IV - complete destruction of CD wall by the offending stone $-4 \%$.
Each type was thought to represent the more advanced form of the previous one. A standardised management protocol was applied to each type and satisfactory results obtained by these authors in the largest reported series so far on MS. This classification appears quite rational and should be acceptable to any surgeon operating on a patient of MS. However because of the limitation that the extents of bile duct erosion which forms the basis of this classification is not amenable to detection on preoperative imaging, the original classification as proposed by Mc Sherry [44] is still popular with surgeons and radiologists who may play an important role in the preoperative management of these patients.

\section{Presenting features}

MS is encountered more commonly in females, probably a reflection of gallstone preponderance in this group of patients. The mean age of presentation has ranged from 21 -90 years in different series [18, 45-46], underscoring the fact that the syndrome is a complication of long standing cholelithiasis. Pain is the most common presenting feature reported in $54-100 \%$ of patients followed by jaundice in $24-100 \%$ and cholangitis in $6-35 \%$ of patients $[11-14,16$, 47]. Charcot's biliary triad is present in $44-71 \%$ of patients $[13,16]$. Presence of painless jaundice may often mimic malignant biliary obstruction. Acute cholecystitis has also been reported in as high as one third of patients $[12,26]$. Less common presentation include acute pancreatitis [16], GB perforation [48] and weight loss [11]. Hepatomegaly is common $[8,11,14,49]$ and a hard GB lump mimicking carcinoma may be palpable in $22 \%$ of patients [14]. Rarely the patient may be completely asymptomatic despite complete erosion of the CD [50]. A high incidence of associated GB malignancy has also been reported [21, 22].

\section{Diagnosis}

Biochemical parameters of liver function show a cholestatic pattern, simulating those of choledocholithiasis. Serum bilirubin ranges from normal to as high as $30 \mathrm{mg} / \mathrm{dl}$ with an average of 7-10 mg/dl. Serum alkaline phosphatase level range from normal to about three to ten fold rise [13-14, 49]. Imaging studies are, however, the mainstay of preoperative diagnosis.

Ultrasound (US) is a good screening method [51], the sensitivity of US in detecting Mirizzi's syndrome is $23-46 \%$ $[18,19]$. Though most often the diagnosis suggested on US is choledocholithiasis only $[13,52]$, the specific finding of this syndrome are, small contracted GB containing stone/s, an echogenic nodule or calculus in the $\mathrm{CD}$, mild to moderate dilatations of the proximal biliary radicals and a normal size or untraced bile duct distal to the calculus or nodule $[13,49,52,53]$. Demonstration of a longitudinal septum 
like structure in the $\mathrm{CD}$, an appearance resulting from the dilated cystic duct running parallel to the bile duct may also point to a diagnosis of MS in suspected cases. Intraductal ultrasound (IDUS) has been reported to be highly sensitive $(97 \%)$ and specific $(100 \%)$ in one study [54].

Computed tomography (CT) when performed may corroborate the US findings [14]. In addition an irregular cavity adjacent to the neck of GB containing the protruding stone is quite characteristic of MS $[52,55,56]$. The duodenum and colon may be seen in close proximity of GB representing bilioenteric fistula, a common occurrence in these patients [52]. CT scanning has a similar sensitivity to US, but can be helpful in diagnosing other causes of obstructive jaundice such as GB cancer, cholangiocarcinoma, or metastatic tumour [56]. Despite the characteristic features, the role of US and CT are mainly to rule out malignancy rather than diagnose MS.

Cholangiography by far remains the most reliable method of diagnosis of this syndrome in the preoperative period. The typical findings on cholangiogram are an eccentric or excavating defect on lateral wall of the $\mathrm{CD}$ at the

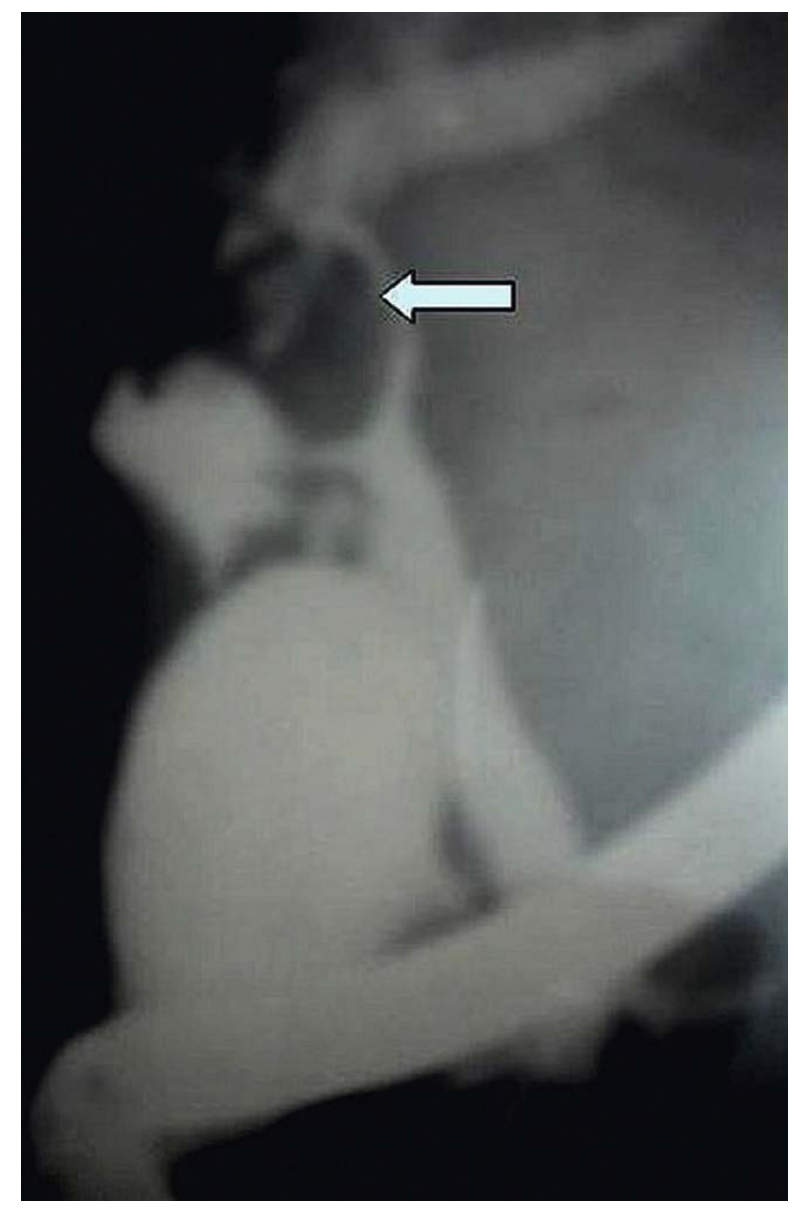

Fig. 1 Endoscopic retrograde cholangiogram showing a large stone (arrow) in the contracted GB protruding into the bile duct causing a concentric filling defect suggestive of Mirizzi syndrome level of the cystic duct or GB neck (Fig. 1). The GB may or may not be visualized. The $\mathrm{CD}$ is dilated proximal to the obstruction and normal distally [4, 6, 27, 42, 52, 57-61]. Similar cholangiographic pictures may also be produced by carcinoma of the cystic duct [17,31] or carcinoma GB [30]. The contour of the filling defect may be irregular in malignancy in contrast to smooth outline of the stone in MS [57]. Endoscopic retrograde cholangiography (ERC) is preferred over percutaneous transhepatic or intravenous cholangiography since in addition to diagnosis it is possible to extract concomitantly bile duct stone/s which may be present in a significant proportion of this patients [11-13, 16, 62-64]. It is also possible to provide preoperative bile drainage in high risk patients by insertion of endoscopic nasobiliary drainage (ENBD) catheter $[13,65]$. The ENBD catheter further helps in identification of CD at surgery [65]. Endoscopic cholangiopancreatography remains the gold standard for the confirmation of the diagnosis; however, magnetic resonance cholangiography (MRC) has been proposed recently as a less invasive, equally effective method of preoperative study [45, 66] (Fig. 2). MRC and ERC are equivalent in their ability to diagnose and delineate details of biliary strictures, and to detect a cholecystocholedochal fistula [19]. In addition, T2 weighted images can differentiate a neoplastic mass from an inflammatory one which may not be possible on US or CT scan [67].

\section{Operative strategy}

In the absence of definite preoperative diagnosis, awareness and careful dissection on the part of the operating surgeon may unmask a few unsuspected cases. The finding of dense

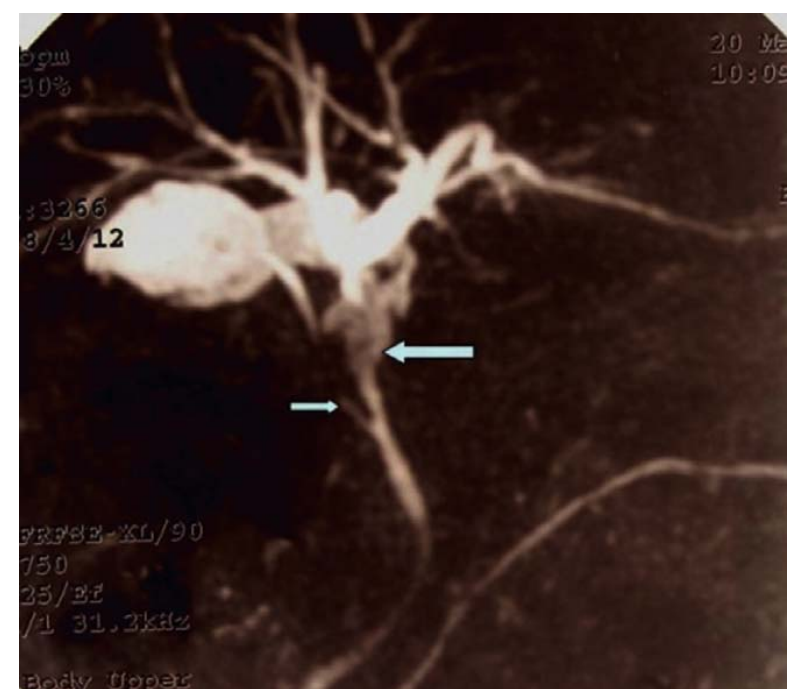

Fig. 2 Magnetic resonance cholangiogram showing stone in the Hartman's pouch (thick arrow) pressing over CBD. Note the low insertion of posterior sectoral duct (thin arrow), requiring extra precaution during surgery 
pericholecystic adhesions, shrunken and sessile GB with/ without cholecystoenteric fistula, obliterated calots triangle should arouse suspicion of this entity [11-14, 49, 65]. The impacted or the eroding stone may simulate carcinoma of the GB neck, thus further complicating the already complicated situation. An intraoperative cholangiogram performed in the early part of surgery confirms the diagnosis and helps in delineating the biliary anatomy [11-13, 39, 41, 49]. An antegrade approach to GB is the uniform recommendation for suspected or confirmed cases [11-14, 49, 65]. The fundus of the GB is opened and the stone/s extracted. The cystic duct stone should be milked back in to the GB $[25,44]$. In rare instances when the Hartman's pouch or cystic duct lies behind the CBD, retrieval of the offending stone may be difficult. A transcholedochal approach has been recommended in such situation [68]. It is often surprising to find the so called growth at the neck of GB disappear with evacuation of stone/s. A frozen section biopsy may be done if concomitant malignancy is strongly suspected. A gush of bile at this stage point to presence of cholecystobiliary fistula [11, 49], assessment of the extent of bile duct erosion which dictates the subsequent management is made at this stage.

In the absence of any bile duct erosion i.e. type I MS, partial cholecystectomy alone is adequate $[12,13,16,23$, 69]. After removal of the offending stone the Hartman's pouch or the parallel cystic duct is left behind. The inflammatory adhesion between these structures and CD precludes any dissection in this area and is bound to result in bile duct injuries if insisted upon. The cystic duct opening in the retained Hartman's pouch is usually in an obliterated state because of repeated inflammation, when present can be closed by a purse string suture around its opening on the mucosal aspect of Hartman's pouch [69].

Presence of CBF calls for a cautious approach. When bile duct erosion is significant late strictures has been reported with simple closure of fistula or end to end repair $[12,15]$. In most of the cases the defect in the CD can be managed by retaining a cuff of GB around the fistula which is approximated, the procedure thus known as choledochoplasty $[11-14,17,39,59]$. The $C D$ in such cases should be drained by a T-tube placed through a fresh choledochotomy or the fistula itself. The tube should be kept for at least two to three months $[11,12]$. Csendes reported an increased incidence of bile leak when the tube was bought out through the fistula rather than a fresh choledochotomy [12].

Though choledochoplasty alone may suffice in nearly all cases of CBF [11], there are patients who present with complete or near complete obstruction of the bile duct at the initial exploration itself $[12,14,49]$. In these cases for better long-term results it is safer to perform bilioenteric anastomosis [15]. The various forms of which could be, hepatico jejunostomy end to side or side to side, cholecystochole-dochoduodenostomy. The other indication for bilioenteric anastomosis could be presence of concomitant multiple CBD stones [13] or distal obstruction of bile duct due to other cause [11, 49].

A well defined management guideline was provided by Csendes et al who classified MS on the basis of extent of erosion of CD circumference. The recommended procedures for different types are: type I - partial cholecystectomy, type II - suture closure of fistula or choledochoplasty, type III - choledochoplasty, type IV - bilioenteric anastomosis. A satisfactory outcome in a mean follow up of 5.7 year is a testimony to the adequacy of these procedures [12]. This management protocol has been followed by other authors too $[13,70]$. Bilioenteric anastomosis has been performed in these series for some of type III case too, where bile duct erosion was considered significant.

Concomitant choledocholithiasis has been reported in $35-62 \%$ of patients with MS [11-13, 16]. Routine exploration of the CD should be carried out in all cases with CBF [70]. For type I, cases one can be more selective. The CD exploration is performed through the fistula itself [11, 14 , $49,70]$ or a fresh choledochotomy $[12,13]$.

\section{Mirizzi syndrome in the laparoscopic era}

This entity was initially considered an absolute contradiction for laparoscopic cholecystectomy [71]. The reason for this could be the sessile GB, frozen calots triangle, the recommended fundus first method, presence of bilioenteric fistula in a sizable amount of patients, and the need to rule out malignancy. Knowingly or unknowingly when it was attempted conversion to open cholecystectomy was required in face of real or threatening bile duct injury [16, 72, 73]. However with further evolution and increasing familiarity, successful management of MS by laparoscopic technique are now being increasingly reported in literature [74-77].

Difficult anatomy in these patients may necessitate insertion of extra ports in addition to the conventional ones [76, 77]. A careful and patient dissection is of paramount importance to delineate the altered anatomy. Lateral traction of the infundibulum of GB, unlike routine cases, does not open up the calots triangle because of dense fibrosis in this area. Special emphasis must be placed on identification of cystic duct - GB and cystic duct - CD junction [16, 72]. Intraoperative cholangiogram $[16,73]$ or laparoscopic ultrasound [77] are of great assistance in these difficult situations. The basic principle of management of various types of MS is akin to that in open surgery. For type I, GB is opened at the fundus, stone evacuated, anterior wall of the GB is excised with diathermy and the Hartmann's pouch is left adherent to the $\mathrm{CD}$. In the single case reports, the authors did not find bile emptying from the GB remnant, thus presuming the cystic duct to be obliterated and no attempt was made to ligate it, the patients made an uneventful recovery [76]. In presence of $\mathrm{CBF}$, partial cholecystectomy by fundus first 
method and choledochoplasty around a T-tube has been performed with a successful outcome [74, 77].

While it appears to be feasible, especially for type I anatomy [45, 78-81], the routine use of laparoscopic surgery as the primary treatment of MS is controversial [82, 83]. It has been suggested, that a prudent approach for type $1 \mathrm{MS}$ is to perform a trial laparoscopic dissection, but to have a low threshold to convert to an open procedure. This approach should be undertaken only by experienced laparoscopic surgeons [79, 82]. More over it is now amply clear that partial cholecystectomy and choledochoplasty alone may be inadequate in presence of significant erosion of $\mathrm{CD}[12,15]$. Bilioenteric anastomosis which is required in these patients is certainly a difficult proportion by laparoscopic technique. Therefore till such time that more experience is available, we would concur with other authors that only type I MS may be tried by laparoscopic method by an experienced surgeon, in a centre with adequate facilities and a low threshold for conversion to open method.

\section{Endoscopic management of Mirizzi syndrome}

In MS conventional methods of endoscopic extraction fails because of the inability to capture the offending stone. However with the availability of modern adjuncts it is now possible to treat some of these patients successfully $[47,84$, 85].

In a report by Binmoeller et al [47], 14 patients with cystic duct stones were selected for endoscopic treatment after being considered unfit for surgery due to various reasons. Using "mother baby scope system" the offending stone/s were visualised and fragmented with the help of electrohydraulic lithotripsy (EHL) introduced through its operating channel. Stone fragments larger than $0.5 \mathrm{~cm}$ in diameter were refragmented and subsequently extracted by dormia basket. The median time required for the entire procedure was 60 minutes. The treatment was successful in all but one patient. In the latter patient asymptomatic leakage of contrast media in to peritoneum was observed from the cystic duct. The patient responded to nasovescicular catheter, intravenous fluid and antibiotics.

Under cholangioscopic control EHL of the offending stone by percutaneous transhepatic approach has also been reported by Cairns et al [84]. Longterm success appears to be most likely in patients with type II disease who do not have residual gallbladder stones [86]. England et al [46] have also shown that endoscopic treatment is an effective temporary measure before surgery and can be a definitive treatment for candidates unsuitable for surgery.

The above reports prove the feasibility of endoscopic treatment abeit in a selective group of patients. The need for sophisticated set up and an experienced endoscopist/ interventionist are the obvious limitation of this approach.

\section{Conclusion}

Presence of MS makes cholecystectomy an extremely hazardous procedure. This syndrome, characterized by bile duct obstruction due to stone in Hartman's pouch or cystic duct is classified in to two types. Obstruction by external compression is classified as type I and that by an eroding stone forming a cholecystobiliary fistula as type II. Both are considered evolving stages of one pathological process. Further classification has been suggested according to the extent of circumferential erosion of common duct. The clinical and biochemical feature of this entity simulate that of choledocholithiasis. Cholangiography remains the mainstay of diagnosis. An eccentric filling defect in common duct at the level of GB with proximally dilated and distally collapsed bile duct suggests MS. Fibrotic contracted GB with dense pericholecystic adhesions, obliterated cystic duct and frozen calots triangle should arouse suspicion of this entity at lapratomy. An antegrade or fundus first method must be undertaken in all suspected or proven cases to avoid bile duct injury. After giving an incision at the fundus gallstone/s is/are evacuated and assessment of the type of MS is made. For type I MS, partial cholecystectomy leaving behind the Hartman's pouch adherent to the bile duct is an adequate procedure. In presence of cholecystobiliary fistula, simple repair of fistula is fraught with danger of long-term stricture formation. Partial cholecystectomy, choledochoplasty utilizing the cuff of the GB and T-tube drainage of the common duct suffices in the majority. Bilioenteric anastomosis is indicated when the bile duct circumference erosion has been considerable. Concomitant choledocholithiasis may be present in up to two third of these patients making common bile duct exploration mandatory. In Mirrizi type I laparoscopic method can be tried but only at well equipped centres for advanced laparoscopy and by an experienced surgeon, for a beginner or a primary set up it should be considered a relative contraindication till more experience is available endoscopic extraction of the offending stone/s has also been tried in a select group of patients in experienced hands with good results.

\section{References}

1. Kher H (1905) Die in neiner Klinik geubte Technik der Gallenstein operation, Munchen J F Lehmann

2. Ruge E (1908) Deitrage zur chirurgischen Anatomie der grossen Gallenwege (Ductus Hepaticus, choledochous and pancreaticus) Arch Clin Chir LXXVII:47

3. Mirizzi PL (1948) Sindrome del conducto hepatico. J Int Chir 8:731-777

4. Clement AR, Lowman RM (1965) The Roentgen features of the Mirizzi syndrome. Am J Roentgenol 94:480

5. Dewbury KL (1979) The features of Mirizzi syndrome on ultrasound examination.Br J Radiol 52:990-992 
6. Koehler RE, Melson GL, Lee JKT, Long J (1979) Common hepatic duct obstruction by cystic duct stones: Mirizzi syndrome. Am J Roentgenol 132:1007-1009

7. Balthazar EJ (1975) The Mirizzi syndrome: inflammatory stricture of the common hepatic duct. Am J Gastroenterol 64:144-148

8. Starling JR, Matallan RH (1980) Benign mechanical obstruction of the common hepatic duct (Mirizzi syndrome). Surgery 88:737-740

9. Ravo B, Epstein H, La Mendolo S, Ger R (1986) The Mirizzi syndrome; preoperative diagnosis by sonography and transhepatic cholangiography. Am J Gastroenterol 81:688-690

10. Singh R, Chawla YK, Katiyar S (1991) Mirizzi syndrome; a rare cause of obstructive jaundice. Trop Gastroenterol 12: 37-39

11. Corlette MB, Bismuth H (1975) Biliobiliary fistula: A trap in surgery of cholelithiasis. Arch Surg 110:377-385

12. Csendes A, Diaz JC Burdiles P, Malueda F, Navo O (1989) Mirizzi syndrome and cholecystobiliary fistula: a unifying classification. Br J Surg 76:1139-1143

13. Ibrarullah MD, Saxena R, Sikora SS, Kapoor VK, Saraswat VA, Kaushik SP (1993) Mirizzi syndrome identification and management strategy. Aus NZ J Surg 63:802-806

14. Rao PSV, Tandon RK, Kapur BML (1988) Biliobiliary fistula: review of nine cases. Am J Gastroenterol 83:652-657

15. Baer HU, Matthews JB, Schweitzer WP, Gertsch P, Blumgart LH (1990) Management of Mirizzi syndrome and the surgical implications of cholecystocholedochal fistula. Br J Surg 77:743-745

16. Curet MJ, Rosendale DE, Congilosi (1994) Mirizzi syndrome in a native American population. Am J Surg 168: 616-621

17. Waisberg J, Corona A, de Abreu IW, Farah JFM, Lupinacci RA, Goffi FS (2005) Benign Obstruction of the Common Hepatic Duct (Mirizzi Syndrome): diagnosis and operative management. Arq Gastroenterol 42:13-18

18. Yeh, CN, Jan, YY, Chen, MF (2003) Laparoscopic treatment for Mirizzi syndrome. Surg Endosc 17:1573-1578

19. Chan CY, Liau KH, Ho CK, Chew SP (2003) Mirizzi syndrome: a diagnostic and operative challenge. Surgeon 1: 273-278

20. Bower TC, Nagorney DM (1988) Mirizzi syndrome. HPB Surgery 1:67-76

21. Prasad TL, Kumar A, Sikora SS, Saxena R, Kapoor VK (2006) Mirizzi syndrome and gallbladder cancer. J HBP Surg 13:323-326

22. Redaelli CA, Buchler MW, Schlling MK, Krahenbuhl L, Ruchti C, Blumgart LH, Baer HU (1997) High coincidence of Mirizzi syndrome and gallbladder carcinoma. Surgery 121:58-63

23. Matthews JB, Blumgart LH (1994) Benign biliary strictures. In Blumgart LH, Ed. Surgery of the liver and Biliary tract. London: Churchill Livingstone 865-895

24. Dietrich KF (1963) Die Hepatikusstenose bei Gallenblasenhals and Zystikussteinen. Brun's Beitr Klin Chir 206:9-22

25. Witte CL (1984) Choledochal obstruction by cystic duct stone. Mirizzi syndrome Am Surg 50:241-243

26. Morelli A, Narducci F, Ciccone R (1978) Can Mirizzi syndrome be classified in to acute and chronic form? Endoscopy 10:109-112
27. Palmer FJ, Goldin GA (1984) The Mirizzi syndrome: a cause of common bile duct obstruction. Australas Radiol 28:26-30

28. Nolan DJ, Espiner HJ (1972) Compression of the common bile duct in acute cholecystitis.Br J Radiol 45:821-825

29. Kumar A, Aggarwal S, Iles SE, Barnes DC (1991) Scintigraphic findings in a case of variant Mirizzi syndrome. Clin Nucl Med 16:912-913

30. Musher DR, Madera MA, Tobias H (1976) Carcinoma of the gallbladder, a diagnosis aided by endoscopic and percutaneous transhepatic cholangiography. Am J Gastroenterol 66:79-83

31. Walker J, Kanzer BF (1982) Carcinoma of the cystic duct mimicking Mirizzi syndrome. Am J Gastroenterol 77: 936-938

32. Montefusco P, Spier N, Geiss AC (1983) Another fact of Mirizzi syndrome. Arch Surg 118:1221-1223

33. Farrer DAT (1951) Carcinoma of the cystic duct. Br J Surg 39:183-185

34. Puestow CB (1942) Spontaneous internal biliary fistula. Ann Surg 115:1043-1054

35. Beherend A, Cullen ML (1950) Cholecystocholedochal fistula: an unusual internal biliary fistula. Am Surg 132: 297-303

36. Mirizzi PL (1952) Les Fistules biliobiliares internes spontanees. J Chir 68:23-28

37. Mallet-Guy P, Roget C, Rodi R (1960) Les fistules cholecystocholedociennes. Lyon Clin 56:231-245

38. Kourias B, Tsopes E (1952) Fistules abiliaresinternes spontanees. J Chir 68:23-28

39. Langer B, Pearson FG (1963) Absent cystic duct: an unusual hazard during cholecystectomy. Can J Surg 6:29-32

40. Patt HH, Koontz AR (1951) Cholecystocholedochal fistula: a report of two cases. Ann Surg 134:1064-1065

41. Sutton JP, Sachatello CR (1967) The confluence stone: a hazardous complication of biliary tract disease. Am J Surg 113: 719-722

42. Cornund F, Grenier P, Belghiti J (1981) Mirizzi syndrome and biliobiliary fistula: roentgenologic appearance. Gastrointest Radiol 6:265-268

43. Yip AWC, Ng WS, Suen WS, Cheng CB (1992) Type II Mirizzi syndrome: diagnosis by endoscopic retrograde cholangiopancreatography. J R Coll Surg Endinb 37:49-50

44. Mc Sherry CK, Ferstenberg H, Virshup M (1982) The Mirizzi syndrome: Suggested classification and surgical treatment. Surg Gastroenterol 1:219-225

45. Gomez D, Rahman SH, Toogood GJ, Prasad KR, Lodge JP, Guillou PJ, Menon KV (2006) Mirizzi's syndrome - results from a large western experience. HPB 8 (6):474-479

46. England RE, Martin DF (1997) Endoscopic management of Mirizzi's syndrome. Gut 40:272-276

47. Binmoellar K, Thanke F, Soehendra N (1993) Endoscopic treatment of Mirizzi syndrome. Gastrointest Endosc 39: $532-536$

48. Ibrarullah MD, Saxena R, Sikora SS, Kapoor VK, Kaushik SP (1992) Unusual gallbladder perforation - definition of a new type. Ind J Gastroenterol 11:170

49. Mishra MC, Vashishtha S, Tandon R (1990) Biliobiliary fistula: preoperative diagnosis and management implications. Surgery 108:835-839 
50. Ibrarullah MD, Prasad KR, Kaushik SP (1995) Asymptomatic Mirizzi syndrome. Trop Gastroenterol 16:32-33

51. Baron RL, Stanley RJ, Lee JKT (1995) Body computed tomography: a prospective comparison of the evaluation of biliary obstruction using computed tomography and ultrasonography. Radiology 145:91-98

52. Becker CD, Hassler H, Terrier F (1984) Preoperative diagnosis of the Mirizzi syndrome: Limitation of sonography and computed tomography. Am J Radiol 143:591-596

53. Dewbury KC (1979) The features of the Mirizzi syndrome on ultrasound examination. Br J Radiol 52:990-992

54. Wehermann T, Riphaus A, Martchenko K, Kokabpick S, Pauka N, Stergiou N, Frenz MB (2006) Intraductal ultrasonography in the diagnosis of Mirizzi syndrome. Endoscopy 38:717-722

55. Pedrosa CS, Casanova R, de la Torre S, Villacorta J (1983) CT findings in Mirizzi syndrome. J Comput Assist Tomogr 7: 419-425

56. Berland LL, Lawson TL, Stanley RJ (1984) CT appearance of Mirizzi syndrome (case report). J Comput Assist Tomogr 8:165-166

57. Eisenberg RL (1990) Filling defects in the bile duct. In: Eisenberg. Ed Gastrointestinal Radiology. Philadelphia: JB LippinCott 805-818

58. Yip AWC, Chow WC, Chan J, Lam KH (1992) Mirizzi syndrome with cholecystocholedochal fistula; preoperative diagnosis and management. Surgery 111:335-338

59. Fan ST, Lau WY, Lee MJR, Wong KP, Wong KK (1985) Cholecystohepatico duodenal fistula; the value of preoperative recognition. Br J Surg 72:743-744

60. Cruz FO, Barriga P, Tocornal J, Burhenne HJ (1983) Radiology of the Mirrizi syndrome: diagnostic importance of the transhepatic cholangiogram. Gastrointest Radiol 8:249-253

61. Tullasay Z (1986) Endoscopic Retrograde Cholangiopancreaticography in Mirizzi syndrome. Am J Gastroenterol 81: 391-392

62. Nagakawa, T, Ohta, T, Kayahara, M et al (1997) A new classification of Mirizzi syndrome from diagnostic and therapeutic viewpoints. Hepatogastroenterology 44:63-67

63. Cotton PB (1984) Endoscopic management of bile duct stones. Gut 25:587-597

64. Siegel JH, Yatto RP (1984) Biliary endoprosthesis for the management of retained bile duct stones. Am J Gastroenterol 79:50-54

65. Dewar G, Chung SCS, Li AKC (1990) Operative strategy in Mirizzi syndrome. Surg Gynecol Obstet 172:157-159

66. Matthews BD, Sing RF, Heniford BT (2000) Magnetic resonance cholangiopancreatographic diagnosis of Mirizzi's syndrome. J Am Coll Surg 190:630

67. Choi BW, Kim MJ, Chung JJ et al (2000) Radiologic findings of Mirizzi with emphasis on MRI. Yonsei Med J 41(1): 144-146

68. Kacker LK, Ravindra KV, Sikora SS, Kapoor VK, Kaushik SP (1994) Transcholedochal cholecystolithotomy for a variant of Mirizzi syndrome.Trop Gastroenterol 15:105-109
69. Ibrarullah MD, Kacker LK, Sikora SS, Saxena R, Kapoor VK, Kaushik SP (1993) Partial cholecystectomy - safe and effective. HPB Surg 7:61-65

70. Martin RF, Rossi R (1994) Bile duct injuries - specimen, mechanism of injury and there prevention. Surg Clin North Am 74:781-803

71. Cuschieri A (1992) The difficult cholecystectomy. In Cuschieri Berica. Ed Laparoscopic biliary surgery. London: Oxford Blackwell Scientific publication, 10

72. Rust KR, Clancy TV, Warren G, Mertesdorf J, Maxwell JG (1991) Mirizzi syndrome: a contraindication to coelioscopic cholecystectomy. J Laparoendosc Surg 1:133-137

73. Posta CG (1995) Unsuspected Mirizzi anatomy: a major hazard to the common bile duct during laparoscopic cholecystectomy. Surgical Laparoscopy and Endoscopy 5: 412-414

74. Binnie NR, Fixoa SJ, Palmer KR (1992) Mirizzi syndrome managed by endoscopic stenting and laparoscopic cholecystectomy. Br J Surg 79:647

75. Paul MG, Burris DG, Mc Guirre AM, Thorfinnson HD, Schonekas H (1992) Laparoscopic surgery in the treatment of Mirizzi syndrome. J Laperoendosc Surg 2:157-163

76. Crosthwaite G, McKay C, Anderson JR (1995) Laparoscopic subtotal cholecystectomy. J R Coll Surg Edinb 40:20

77. Meng WCS, Kwok SPY, Kelly SB, Lau WY, Li AK (1995) Management of Mirrizi syndrome by laparoscopic cholecystectomy and laparoscopic ultrasonography. Br J Surg 82:396

78. Kelly MD (2005) Mirizzi Syndrome. ANZ J Surg 75: 501-503

79. Vezakis A, Davides D, Birbas K et al (2000) Laparoscopic treatment of Mirizzi syndrome. Surg Endosc 10(1):15-18

80. Chowbey PK, Sharma A, Mann V, Khullar R, Baijal M, Vashistha A (2000) The management of Mirizzi syndrome in the laparoscopic era. Surg Laparosc Endosc Percutan Tech 10:11-14

81. Kwon AH, Inui H (2007) Preoperative diagnosis and efficacy of laparoscopic procedures in the treatment of Mirizzi's syndrome. J Am Coll Surg 204(3):409-415

82. Targarona EM, Andrade, E, Balague, C et al (1997) Mirizzi's syndrome. Diagnostic and therapeutic controversies in the laparoscopic era. Surg Endosc 11:842-845

83. Sare M, Gurer S, Taskin V et al (1998) Mirizzi's syndrome: Choice of surgical procedure in the laparoscopic era. Surg Laparosc Endosc 8:63-67

84. Cairns SR, Watson CN, Lees WR, Salmon PR (1987) Percuta-neous lithotripsy and endoprosthesis a new treatment for obstructive jaundice in Mirizzi's syndrome. BMJ 295:1448

85. Binmoeller KF, Bruckner M, Thonke F, Soenhendra N (1993) Treatment of difficult bile duct stones using mechanical, electrohydraulic and extracorporal shock wave lithotripsy. Endoscopy 25:201-206

86. Tsuyuguchi T, Saisho H, Ishihara T et al (2000) Long-term follow up after treatment of Mirizzi syndrome by peroralcholangioscopy. Gastrointest Endosc 52:6390-644 